\title{
Flour pasting properties define consumer selection decisions for boiled and flour-based cassava food products in Uganda
}

\author{
Ephraim Nuwamanya 1,3*, Michael Kanaabi ${ }^{1}$, Enoch Wembabazi ${ }^{1}$, Nicholas Muhumuza ${ }^{13}$, Alfred Ozimati ${ }^{1}$, Wil- \\ liams Esuma 1, Paula Iragaba 1, Steven Angudubo 1, AnnRitah Nanyonjo ${ }^{1}$, Hale Tufan ${ }^{2}$ and Robert Kawuki ${ }^{1}$ \\ 1 National Crops Resources Research Institute (NaCRRI), P.O Box 7084, Kampala-Uganda; \\ 2 Cornell University, Plant Breeding and Genetics Section, School of Integrative Plant Science, Cornell Univ., \\ Ithaca, NY, 14853 USA; hat36@cornell.edu \\ 3 Makerere University, College of Agriculture and Environmental Sciences, School of Agricultural Sciences, \\ P.O Box 7062, Kampala, Uganda \\ * Correspondence: nuwamanyaephraim@gmail.com; Tel.: +256-771881992
}

\begin{abstract}
Enhanced adoption of new cassava varieties is dependent on the ability to breed for acceptability attributes that define boiled roots and flour-based meals. This study assessed the role of pasting properties in defining farmers' acceptability. Cassava accessions in a Triadic Comparison of Technologies (TRICOT) approach were assessed for acceptability in addition to moisture content root softness (boiled roots)and pasting properties (flour). Results showed location based differences in moisture content (13-17\%) and boiled root softness (0.5-6.0 N/cm). Pasting properties such as peak viscosity (4300-5500 cP), breakdown (2204-3234 cP) and final viscosity (2953-3493) also varied significantly among accessions. Linear relationships were observed between consumer acceptance parameters and pasting properties \{(final viscosity and root mealiness ( $r=0.7$ ), ease of mingling $(r=0.6)$, product stickiness $(r=0.5)$ and overall performance of the flour based meal $(\mathrm{r}=0.6)\}$. Therefore pasting property analysis can differentiate cassava accessions and is an important tool in selection of consumer acceptable varieties.
\end{abstract}

Keywords: cassava, pasting properties, acceptability, consumer preference, selection metrics.

\section{Introduction}

Cassava's (Manihot esculenta Crantz) ever-increasing role in food and industrial value chains justifies interventions aimed at sustaining and/or increasing its production and productivity [1]. It's partly for this reason that significant efforts and resources have for the past decades been devoted to genetically improve cassava and enable it overcome acute production stresses and/or obstacles to optimal production [2]. These breeding efforts led to increased adoption of new varieties i.e., adoption increasing from 20\% in 1993 to $80 \%$ in 1999 and is still increasing up to now [3].

However, some improved varieties like NASE1, NASE10, NASE 11 have since been abandoned with farmers choosing to revert back onto their local varieties which are known to have good root quality traits despite having poor agronomic performance[4][5]. Consequently, this has been a major a major challenge to cassava breeding's relevance and thus necessitating a redesign of the breeding process and philosophy. Worse still is the hazy description of preferred end-user traits which makes it difficult to measure and henceforth to breed for [6]. In some instances, a specific end-user need is tagged to a range of traits which if not well understood cannot be bred and/or selected [3]. On the other hand, some traits related to the processed product and/or the processing conditions, are definitive of the acceptability of a particular product from a variety [7]. Such traits may not be readily measured in the raw primary product.

Traits such as the cooking time, boiled root quality, compositional profiles of processed flour, the cooking properties and their interactions, drive 
acceptability[8][9][10][11]. Leveraging on lessons elsewhere, preferred and non-preferred quality traits in raw, processed and final-end product have been compiled in Uganda to inform breeding decisions [12]. However, before prioritizing such traits, it is important to know how these traits are measured, and understand the relationships between such traits and sensorial properties upon which selection decisions are made. With some traits being associated with gender and/or group-based livelihood strategies[12], the inclusion of such traits in variety development is increasingly becoming critical.

Triadic comparisons of technologies (TRICOT) provides for a farmer-led pro-active approach that enables inclusive and unbiased participation of farmers in variety selection decisions[7]. TRICOT allows the interrogation of farmer preferences and enables each farmer to identify the 'best' and 'worst' variety in aspects of agronomy, processing, culinary traits and overall performance conditions. Thus in this study, we adopted the TRICOT approach in evaluating the food products arising from our elite cassava accessions. Specifically, the objective of this study was to determine the pasting properties of flour obtained from farmers participating in TRICOT trials alongside farmer led acceptability assessments of the accessions used. This was meant to provide a definitive understanding of pasting properties as measurable traits that define consumer preferences for boiled cassava roots and flour-based meal in Uganda. The influence of these traits on acceptability of a particular variety among farmers was also assessed.

\section{Materials and Methods}

\subsection{Study area description}

This study targeted regions were "boiled roots" and/or "flour-based meal" are primary cassava food products consumed by residents[7]. Accordingly, for 'flour-based meal" the districts of Serere and Kaberamaido (eastern region), and Arua (West Nile region) were selected. For "boiled roots" four districts namely Luwero and Mityana (central region), and Serere and Kaberamaido (Eastern region), were selected. Thus, Serere and Kaberamaido served as comparative locations for both cassava products under assessment. In each of these districts, farmer managed variety selection trials were established following the TRICOT procedure where randomization is maximized among farmers. Briefly, two counties leading in cassava production were selected per district with help of district agricultural officers. From each county, four parishes were selected while 10 farmers were selected per parish. Each farmer was randomly assigned three accessions coded as " $\mathrm{A}$ " "B" and " $\mathrm{C}$ ". These were established on small plots that comprised 18 plants planted in three-meter rows of six plants per row. Farmers were encouraged to maintain their plots weed-free throughout the experimentation period that lasted 12 months when harvesting and processing of food products was done.

\subsection{Consumer's acceptability tests on "boiled roots"}

At each farmers field (Mityana, Luwero Arua and Kaberamaido and Serere) five plants were harvested per accession. All roots harvested from each plot were separately placed in plastic bags labelled "Plot A" "Plot B" and "Plot C" and carried to the farmer's home. Three appealing roots were then selected per accession, peeled and washed. Tapering ends of the root (proximal and distal) were chopped off and the remaining section peeled, washed in clean water and sectioned into small pieces according to routine farmer's practices.

Thereafter, the chopped pieces were separately placed in three saucepans labelled "Plot $\mathrm{A}^{\prime}$ "Plot B" and "Plot C". The pans were covered with banana leaves and cooking was then done concurrently over firewood. To allow for fair comparison of accessions, three fire places were lit concurrently as done routinely by farmers. Cooking was done for 45 
minutes. After 45 minutes, the boiled roots were served and assessed for sensory and softness attributes.

Accordingly, each farmer assessed boiled root acceptability of the three accessions harvested at his/her site for cooking time, mealiness, taste, softness, fibrousness and overall liking[12][7]. This assessment involved defining the "best" and "worst" accessions per attribute as outlined in TRICOT methodology. Furthermore, each accession was assessed for softness using penetrometer [12]. The probe of the penetrometer was pressed into each cooked piece to a depth of $1 \mathrm{~cm}$ and the force recorded in $\mathrm{N} / \mathrm{cm}$. Twelve (12) readings were recorded per accession.

\subsection{Consumer's acceptability tests on "flour-based meal"}

From the roots harvested in the districts of Kaberamaido, Serere and Arua, assessment of flour product was done alongside boiled cassava. At least 2-3 marketable roots were picked and approximately three-quarters of the total roots harvested per plot were peeled, washed, sliced into thin sections according to the farmer's routine practice. The slices were dried for three days. The dried chips were then milled into flour per plot labelled as " $\mathrm{A}$ ", " $\mathrm{B}$ " or " $\mathrm{C}$ ". The acquired flour was then prepared into cassava flour based meal. Briefly, the farmer boiled enough water to make three "flour-based meals" tagged to each of the plots A, B and C. Once the water boiled, the household member entrusted with preparing meals took turns to make a meal from each accession, ensuring that similar quantity $(1 \mathrm{~kg})$ of flour and water was used for each accession. The prepared "flour-based meal" was then served on three separate pre-labeled plates.

Acceptability assessments of "flour" by farmers were based on ease of flour drying and ease of mingling. The "flour-based meal" was also assessed for color, texture and taste. Just like for boiled roots, individual farmer's ranked "best" and "worst" accessions. Data from different farmers was then aggregated on a 10 point scale as a description of each parameter and average scores of each variety across different farmers were generated.

Remnant flour samples tagged to respective plots ("Plot A" "Plot B" and "Plot C") from farmers were shipped to the laboratory for further analysis. Firstly, assessment was made for moisture content using a modified procedures [13]. Secondly, assessment for flour pasting properties was undertake using a rapid viscosity analyzer (RVA-4500, Perten Instruments, Australia) and the Thermocline for Windows Software, using a modified flour profile as described. For pasting properties, three grams of flour were weighed up into the RVA canister and a volume equivalent to $25 \mathrm{~g}$ of distilled water added. The RVA test profile was held constant at $50{ }^{\circ} \mathrm{C}, 960 \mathrm{rpm}$ mixing speed for 10 seconds. The mixing speed was decreased to $160 \mathrm{rpm}$ and the temperature held at $50{ }^{\circ} \mathrm{C}$ for an extra $50 \mathrm{sec}-$ onds. The temperature was then steadily increased to $95^{\circ} \mathrm{C}$ within 4 minutes and held constant at $95^{\circ} \mathrm{C}$ for $2.5 \mathrm{~min}$ and steadily decreased back to $50{ }^{\circ} \mathrm{C}$ in 4 minutes. The final viscosity was documented after 13 minutes. This analysis enabled quantification of flour viscous load on pasting; flour ability to withstand heating and shear stress; time to attain peak viscosity; flour process-ability temperature; degree of re-association of pasted flour during cooling and tendency to formation of gel after cooking.

\subsection{Data analysis}

Data on acceptability of "boiled roots" was aggregated on the basis of accessions and the districts in which those accessions were grown. For each of the districts, a summary of performance of each accession was generated for each farmer. With each farmer growing three selected accessions, the best ranked accession for the farmer was assigned "score" three (3) while the worst ranked accession was assigned "score" one (1). Accessions ranked as medium in performance were thus assigned "score" two (2). The assigned 
scores were then used to select the best and worst performing accessions based on the ranking by farmers. Similarly, data on acceptability of "flour-based meal" was also aggregated as for the boiled roots according to the district of origin. Each farmer was allowed to identify the best and worst performing accessions based on the ranks. These ranks were later converted into "score" by assigning "score" three (3) to the best ranked, "score" two (2) to the medium performing accession and "score" one (1) to the worst ranked. Further, regression analyses were conducted to test relationships between: a) softness assessment using penetrometer and sensory attributes of "boiled roots" and "flour-based meal"; and b) pasting properties generated from RVA and acceptability attributes. Correlations between pasting properties, accession performance attributes (scores) and sensory attributes (scores) were derived using the cor-function using R statistical software.

\section{Results}

The results obtained from the various analyses conducted are described. They point out the specific differences on comparison among the accessions used in this study. Results also point out the performance of the different accessions in relation to NAROCASS 1 the best variety of choice among farmers at the moment.

\subsection{Cassava flour moisture content:}

The moisture contents of flour samples prepared by farmers in the study areas ranged from $11-17 \%$. There were no significant variations $(p>0.05)$ observed in accessions of cassava used (Table 1). Accessions MM16/0707 registered the highest moisture contents (15.1\%) while accession UG120024 registered the lowest moisture contents (13.75\%) compared to other accessions and across all the study sites. When study sites were compared, it was observed that farmers in Arua had a lower average moisture content of $13.7 \%$ compared to farmers in Kaberamaido (15.02\%) and Serere (14.53\%). The moisture contents were higher than the recommended for cassava flour in all cases[14][9]. 
Table 1: Flour moisture content of accessions produced from different districts

\begin{tabular}{lllll}
\hline Accession & Arua (\%) & Kaberamaido (\%) & Serere (\%) & Overall average (\%) \\
\hline MM06/123 & $13.35 \pm 1.33$ & $14.47 \pm 1.43$ & $14.24 \pm 1.09$ & $14.02 \pm 0.59$ \\
MM16/0707 & $13.66 \pm 0.66$ & $15.98 \pm 0.95$ & $15.61 \pm 1.27$ & $15.08 \pm 1.25$ \\
MM16/1627 & $13.70 \pm 0.64$ & $15.64 \pm 1.18$ & $14.29 \pm 0.78$ & $14.54 \pm 0.99$ \\
NAROCASS 1 & $13.51 \pm 0.71$ & $15.26 \pm 1.56$ & $14.09 \pm 1.01$ & $14.28 \pm 0.89$ \\
UG120024 & $13.63 \pm 0.36$ & $14.75 \pm 1.12$ & $12.87 \pm 1.58$ & $13.75 \pm 0.95$ \\
UG120124 & $13.63 \pm 0.87$ & $15.88 \pm 1.96$ & $14.59 \pm 1.23$ & $14.70 \pm 1.13$ \\
UG120156 & $14.46 \pm 1.29$ & $15.32 \pm 1.67$ & $13.87 \pm 1.59$ & $14.55 \pm 0.73$ \\
UG120180 & $13.44 \pm 0.75$ & $13.30 \pm 1.01$ & $15.09 \pm 1.32$ & $13.94 \pm 1.00$ \\
UG120193 & $13.66 \pm 0.50$ & $13.68 \pm 1.35$ & $14.53 \pm 1.25$ & $13.96 \pm 0.49$ \\
UG120198 & $13.71 \pm 0.59$ & $15.90 \pm 0.90$ & $14.82 \pm 1.41$ & $14.81 \pm 1.09$ \\
UG130007 & $14.00 \pm 0.86$ & $14.91 \pm 0.98$ & $15.60 \pm 1.22$ & $14.83 \pm 0.80$ \\
UG130016 & $13.63 \pm 0.53$ & $15.18 \pm 0.82$ & $14.74 \pm 0.54$ & $14.52 \pm 0.79$ \\
Average/district & $\mathbf{1 3 . 7 0 \pm 0 . 3 0}$ & $\mathbf{1 5 . 0 2} \pm \mathbf{0 . 9 0}$ & $\mathbf{1 4 . 5 3 \pm 0 . 7 9}$ & $\mathbf{1 4 . 4 2 \pm 0 . 4 4}$ \\
\hline
\end{tabular}

\subsection{Softness of boiled roots:}

Cassava root softness varied significantly across accessions and locations ranging from $1.97 \mathrm{~N} / \mathrm{cm}$ for accession UG120198 to $3.41 \mathrm{~N} / \mathrm{cm}$ in accession UG110017. Generally, cassava accessions in Kaberamaido had lower root softness values (average 1.75N/cm) that were significantly different from other districts of Mityana, Serere and Luwero (average $2.70 \mathrm{~N} / \mathrm{cm}$ ). Overall, the best performing accession for softness was UG120198 and UG120024 while accession UG110017 performed poorly for softness across locations.

Table 2: Variation in softness across trial sites for boiled cassava roots in different districts

\begin{tabular}{llllll}
\hline Clone ID & Mityana & Serere & Luwero & Kaberamaido & $\begin{array}{l}\text { Accession } \\
\text { average }\end{array}$ \\
\hline MM160707 & $2.43 \pm 0.83$ & $2.82 \pm 0.85$ & $2.62 \pm 0.74$ & $1.68 \pm 0.56$ & $2.39 \pm 0.50$ \\
MM06/123 & $2.61 \pm 0.16$ & $2.25 \pm 0.15$ & $2.82 \pm 0.52$ & $1.75 \pm 0.61$ & $2.36 \pm 0.47$ \\
UG110017 & $2.67 \pm 0.42$ & $5.95 \pm 0.11$ & $2.75 \pm 0.48$ & $2.27 \pm 0.49$ & $3.41 \pm 1.70$ \\
UG120024 & $3.36 \pm 0.77$ & $2.04 \pm 0.69$ & $2.80 \pm 0.45$ & $0.73 \pm 0.25$ & $2.23 \pm 1.13$ \\
UG120124 & $2.92 \pm 0.53$ & $3.65 \pm 0.00$ & $2.21 \pm 0.66$ & $1.28 \pm 0.48$ & $2.52 \pm 1.02$ \\
UG120156 & $3.29 \pm 0.74$ & $2.60 \pm 0.03$ & $3.25 \pm 0.54$ & $2.68 \pm 0.74$ & $2.95 \pm 0.37$ \\
UG120180 & $2.29 \pm 0.31$ & $2.82 \pm 1.01$ & $2.60 \pm 0.37$ & $2.07 \pm 0.27$ & $2.45 \pm 0.33$ \\
UG120193 & $2.94 \pm 0.31$ & $2.25 \pm 0.81$ & $2.93 \pm 0.39$ & $2.05 \pm 0.43$ & $2.54 \pm 0.46$ \\
UG120198 & $2.23 \pm 0.34$ & $1.58 \pm 0.18$ & $2.66 \pm 0.47$ & $1.38 \pm 0.61$ & $1.96 \pm 0.59$ \\
UG130007 & $1.92 \pm 0.40$ & $4.10 \pm 0.54$ & $2.71 \pm 0.51$ & $1.39 \pm 0.39$ & $2.52 \pm 1.18$ \\
UG130016 & $2.14 \pm 0.47$ & $1.10 \pm 0.44$ & $2.75 \pm 0.51$ & $1.94 \pm 0.62$ & $1.98 \pm 0.68$ \\
\hline District average & $2.62 \pm 0.47$ & $2.83 \pm 1.34$ & $2.73 \pm 0.25$ & $1.75 \pm 0.54$ & \\
\hline
\end{tabular}




\subsection{Acceptability rankings of "boiled roots" and "flour-based meals" cassava products}

Results for acceptability of the flour based meal and boiled roots are presented in Table 3 . For the flour based meal, the overall best ranked accession was UG120193 followed by NAROCASS 1. The worst ranked accession over all was UG130016. Location specific differences were also observed for the flour meal based product with best ranked accession in Serere being UG120193 while it was UG120156, UG120193 and UG120198 in Kaberamaido. On the other hand, the best ranked accession for the flour based meal in Arua was UG120193 (Table 3)

Over all, the best ranked varieties for boiled root product was UG120193 and UG120124 while the worst ranked varieties were MM16/1627, MM06/123, MM16/0707 and UG130016. In the individual districts, ranking was also different with the NAROCASS 1 as the best ranked variety in Serere, UG120156 as the best in Mityana and Kaberamaido while in Arua it was UG120193. These differences show differences in preference for the boiled root cassava product in specific areas(Teeken et al. 2018).

Table 3: Acceptability scores of different varieties for flour and boiled roots in different districts

\begin{tabular}{lllll|lllll}
\hline \multicolumn{2}{l}{ Flour based meal } & \multicolumn{7}{c}{ Boiled roots } \\
Clone & Serere & Kaberamaido & Arua & Overall & Serere & Mityana & Kaberamaido & Arua & Overall \\
\hline UG120156 & 1 & 5 & 4 & 3.33 & 1 & 3 & 6 & 1 & 2.75 \\
UG120124 & 3 & 2 & 4 & 3 & 0 & 0 & 5 & 4 & 4.5 \\
UG120180 & 3 & 3 & 2 & 2.67 & 1 & 2 & 5 & 3 & 2.75 \\
UG120193 & 5 & 5 & 7 & 5.67 & 3 & 2 & 4 & 10 & 4.75 \\
UG120198 & 2 & 5 & 2 & 3 & 1 & 1 & 3 & 2 & 1.75 \\
UG120024 & 3 & 1 & 0 & 2 & 1 & 0 & 3 & 0 & 2 \\
UG130016 & 1 & 2 & 0 & 1.5 & 1 & 0 & 2 & 0 & 1.5 \\
UG130007 & 4 & 3 & 5 & 4 & 2 & 1 & 2 & 6 & 2.75 \\
NAROCASS 1 & 7 & 4 & 2 & 4.33 & 4 & 2 & 2 & 3 & 2.75 \\
MM16/0707 & 3 & 0 & 2 & 2.5 & 1 & 0 & 2 & 1 & 1.33 \\
MM16/1627 & 1 & 3 & 0 & 2 & 1 & 0 & 1 & 0 & 1 \\
MM06/123 & 3 & 4 & 0 & 3.5 & 3 & 2 & 0 & 1 & 1.5 \\
\hline
\end{tabular}

Note: The value zero (0) denotes that the sample was not assessed in that particular instance.

Location wide comparisons for the performance of the boiled root showed that the most preferred accession over all was UG120193 followed by NAROCASS1 and UG120156. The two accessions (i.e., UG120193 and UG120156) can therefore be considered as replacement varieties for NAROCASS 1 the bench mark variety in this case. In the case of the flour based meal, accessions UG120193, UG130007 and UG120156 and NAROCASS 1 were the most acceptable. In this case too, the accessions UG120193, UG130007 and UG120156 can be considered to replace NAROCASS 1 (Figure 1). 


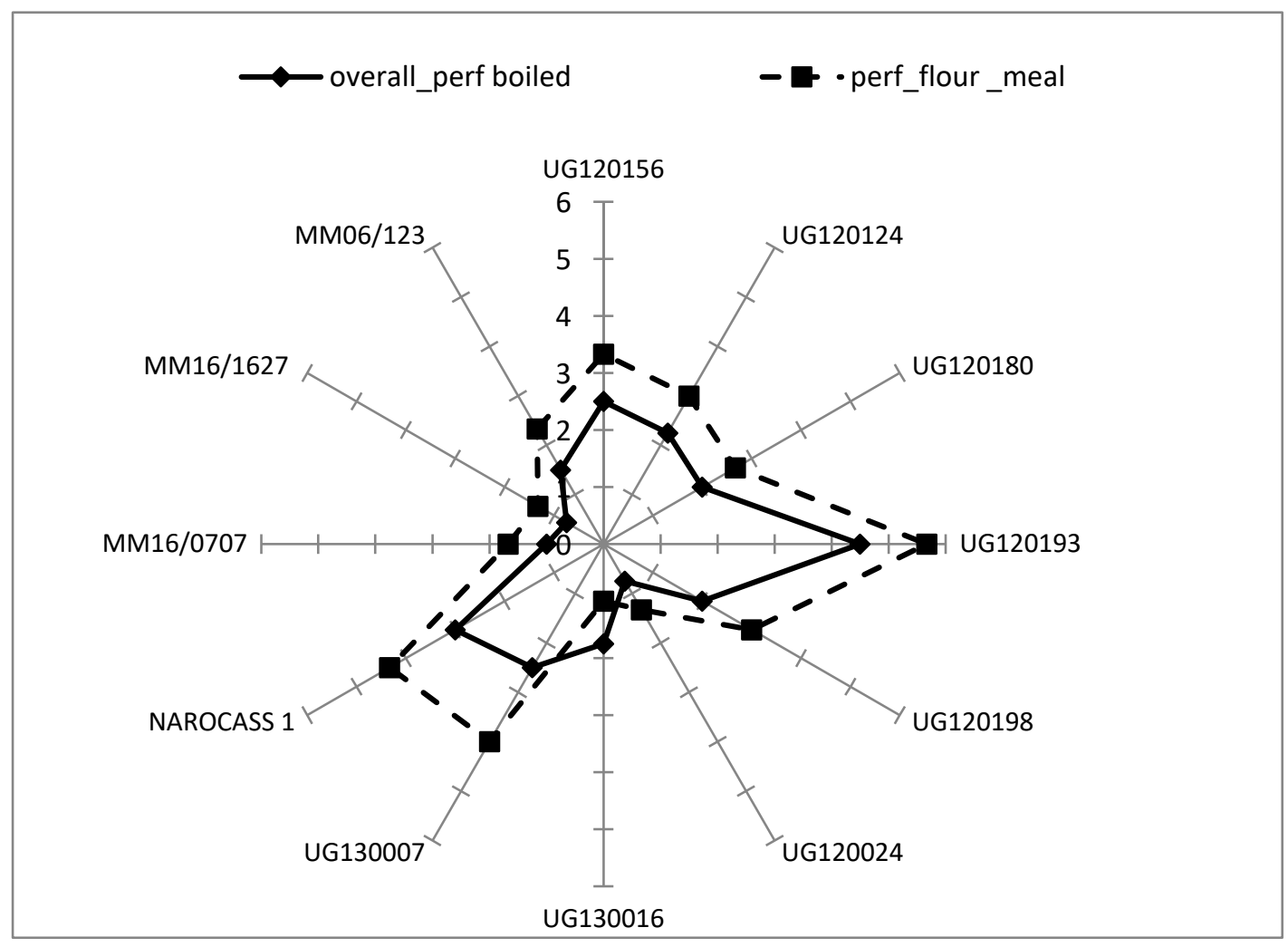

Figure 1: Radar plot showing the overall acceptability of the different accessions based on identified farmer acceptability criteria

\subsection{Pasting properties of cassava flour}

The definitive behavior of cassava flour during pasting was established as presented Table 4 and in Figure 2. Variability within specific accessions grown in different places and used in the study was low much as variety to variety comparisons showed significant differences. Flour peak viscosity ranged from $4300-5600 \mathrm{cP}$ representing a difference of $1200 \mathrm{cP}$ where the reference variety NAROCASS1 with a peak viscosity of $5100 \mathrm{cP}$ was in the mid-range and lower than for accessions UG120124 and UG120198. These variations underpin differences in flour components during cooking[8][9].

Trough viscosity, an indicator of the ability of "flour-based meal" not to collapse during cooking[15], ranged from 2000-2720cP (Table 4). This indicated the differences in holding strength of flour during mingling of the "flour-based meal" for the accessions studied. Breakdown viscosity on the other hand ranged from $2200 \mathrm{cP}$ to $3250 \mathrm{cP}$ and thus being a highly variable quality parameter. In this study, accession UG120156 had the lowest breakdown viscosity while accession UG120198 was the highest, implying that accession UG120198 had the most stable paste. Accessions with high breakdown viscosity have critical usability and process-ability characteristics as has been obeserved in cassava [9] and other flour sources [15][16][17] making them important for use where stable pastes are required. 


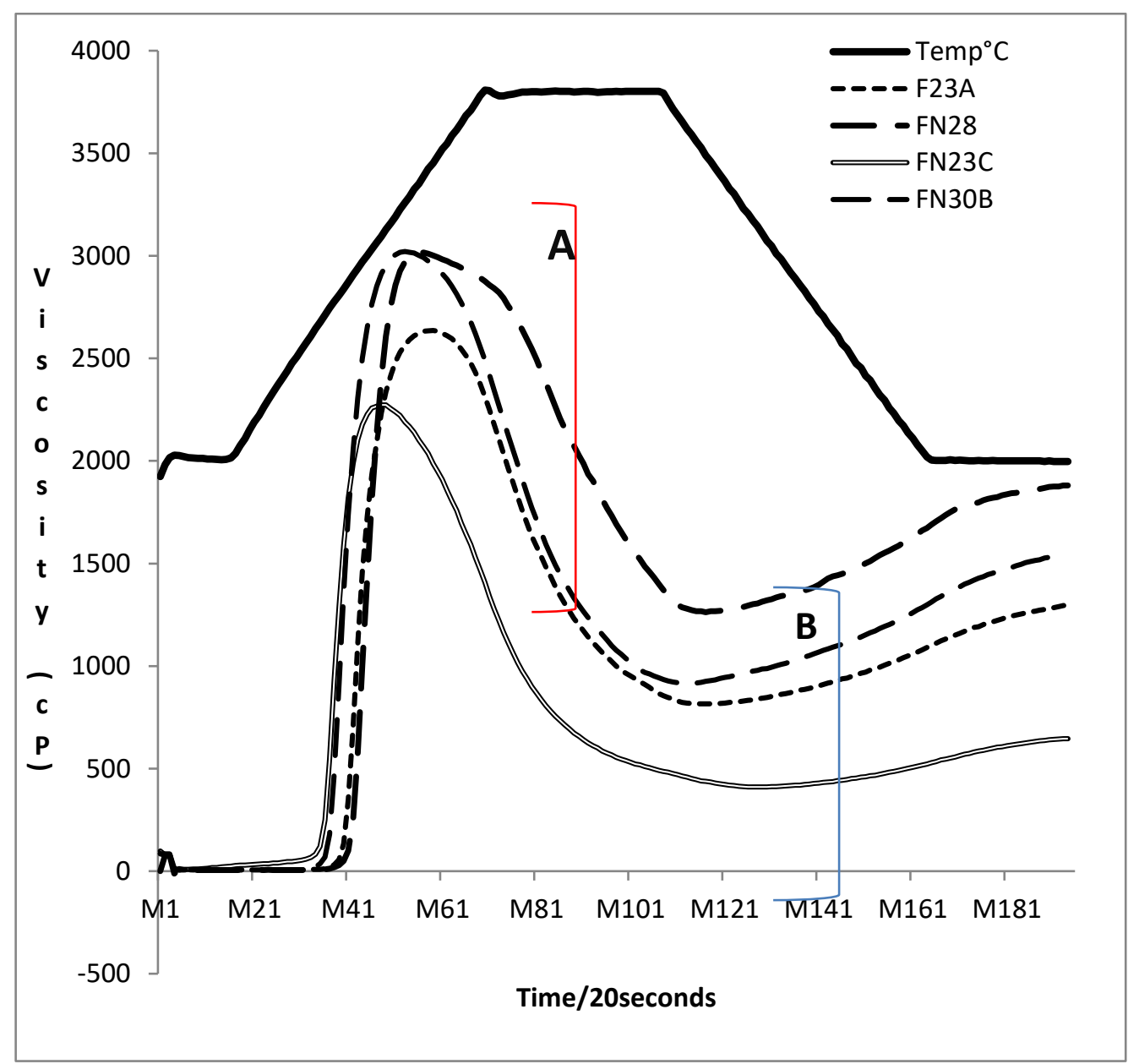

Figure 2: Typical cassava pasting curve showing accession differences in viscosity properties and times to attain peak viscosity. $\mathrm{A}=\mathrm{Observed}$ differences in peak viscosity and times to attain peak; $\mathrm{B}=$ Observed differences in pasting behavior during the cooling phase; $\mathrm{C}=$ Observed differences in final viscosity

In this study, final viscosity ranged from $3000 \mathrm{cP}$ to $3500 \mathrm{cP}$ with clear differences between most of the accessions. The final viscosity shows the restructuring of starch molecules within the paste after cooling [15]. Thus different accessions used in this study restructed differently after cooling producing different characteristic pasting profiles. Like the final viscosity, set back viscosity measures the properties of the final product after cooling and is thus highly related to acceptability criteria of the flour based meal and the boiled root based meals. In this study, the setback viscosity ranged from $820 \mathrm{cP}$ to $1120 \mathrm{cP}$ in the different accessions, showing significant variations $(\mathrm{p}<0.05)$ among accesions. Pasting temperature is associated with onset of starch gelatinization and thus describes temperature for maximum absorption of water by the starch[11]. In this study, pasting temperatures ranged from $70.3-73.3^{\circ} \mathrm{C}$. Since high pasting temperatures are indicative of starch resistance[10][18], accessions such as UG120024, MM16/1627, MM16/0707 with temperatures $>72^{\circ} \mathrm{C}$ would be difficult to process into pastes compared to accessions . The peak time for the accessions used in this study ranged from 3.9-5.1 minutes and was particularly high for accessions with higher pasting temperatures. High peak times were observed for accesion UG120024 (4.3-5.1 minutes) while low peak times were observed for accesion MM06/123 (3.9-4.4 minutes). Peak time describes the time taken to achieve the peak viscosity while heating at $95^{\circ} \mathrm{C}[10][15]$. 
Table 4: Summary results for Rapid Viscosity Analysis of accessions aggregated from the study

\begin{tabular}{|c|c|c|c|c|c|c|c|c|}
\hline Variety/Clone & Peak-Viscosity(00s) & $\begin{array}{l}\text { Trough } \\
\text { ty(00s) }\end{array}$ & Viscosi- & $\begin{array}{l}\text { Breakdown } \\
\text { Viscosity (00s) }\end{array}$ & $\begin{array}{l}\text { Final } \\
\text { ty(00s) }\end{array}$ & $\begin{array}{l}\text { Setback Viscosi- } \\
\text { ty(00s) }\end{array}$ & Peak Time & Pasting Temp \\
\hline MM06/123 & $47.03 \pm 6.64$ & $21.68 \pm 3.17$ & & $27.44 \pm 4.39$ & $30.07 \pm 3.35$ & $10.48 \pm 1.55$ & $4.15 \pm 0.34$ & $71.80 \pm 1.48$ \\
\hline MM16/0707 & $45.82 \pm 6.37$ & $20.80 \pm 3.25$ & & $27.31 \pm 4.61$ & $29.53 \pm 3.19$ & $11.03 \pm 1.84$ & $4.19 \pm 0.38$ & $72.28 \pm 1.01$ \\
\hline MM16/1627 & $48.40 \pm 4.44$ & $24.69 \pm 4.44$ & & $25.95 \pm 4.01$ & $32.21 \pm 4.17$ & $9.77 \pm 1.56$ & $4.46 \pm 0.27$ & $72.70 \pm 0.91$ \\
\hline NAROCASS1 & $51.36 \pm 5.87$ & $27.20 \pm 5.16$ & & $26.76 \pm 5.63$ & $34.93 \pm 5.36$ & $10.33 \pm 1.95$ & $4.41 \pm 0.37$ & $71.36 \pm 1.14$ \\
\hline UG120024 & $43.94 \pm 4.62$ & $22.78 \pm 4.13$ & & $22.04 \pm 1.95$ & $30.92 \pm 3.97$ & $9.02 \pm 1.12$ & $4.64 \pm 0.36$ & $73.31 \pm 0.88$ \\
\hline UG120180 & $48.26 \pm 3.62$ & $22.68 \pm 4.01$ & & $27.53 \pm 3.44$ & $29.65 \pm 4.15$ & $8.92 \pm 1.74$ & $4.26 \pm 0.31$ & $71.23 \pm 1.07$ \\
\hline UG120193 & $51.59 \pm 7.13$ & $25.76 \pm 3.99$ & & $27.82 \pm 5.53$ & $33.62 \pm 3.25$ & $9.86 \pm 1.76$ & $4.46 \pm 0.37$ & $71.84 \pm 1.49$ \\
\hline UG120198 & $56.80 \pm 4.98$ & $25.76 \pm 3.38$ & & $32.34 \pm 4.77$ & $32.68 \pm 2.91$ & $8.23 \pm 1.81$ & $4.31 \pm 0.29$ & $71.00 \pm 0.67$ \\
\hline UG130007 & $49.11 \pm 7.41$ & $24.48 \pm 3.52$ & & $26.79 \pm 4.94$ & $32.06 \pm 4.05$ & $9.74 \pm 1.58$ & $4.36 \pm 0.35$ & $70.79 \pm 1.32$ \\
\hline UG130016 & $48.27 \pm 6.41$ & $23.05 \pm 3.36$ & & $26.85 \pm 4.93$ & $30.83 \pm 3.42$ & $9.41 \pm 2.08$ & $4.39 \pm 0.46$ & $70.27 \pm 0.96$ \\
\hline
\end{tabular}




\subsection{Relationship between "flour-based meal" acceptability and pasting properties}

Selection of the best "flour-based meal" from the evaluated accessions would ultimately depend on consumers and/or farmer's preferences[15][3], which is very likely to be subjective and highly variable among households[3]. Thus in this study, we hypothesized that through quantifying pasting properties we could identify a metric that can corroborate consumers and/or farmer's preferences for "flour-based meal" product. Thus relationships between flour based meal acceptability parameters and the pasting properties were assessed using correlation analysis.

Peak viscosity had a positive significant relationship with "flour-based meal" stickiness $(\mathrm{r}=0.5, \mathrm{p}=0.05)$, and with overall performance $(\mathrm{r}=0.5, \mathrm{p}=0.05)$ showing that "flour-based meal" stickiness can be quantified using peak viscosity as a metric. As such, high peak viscosity was observed in accessions UG120124, UG120193, UG120198 which also had high stickiness rankings. However, weak negative correlations $(r=-0.2, p=0.1)$ were observed between peak viscosity and "ease of mingling" as it would be hard to mingle a very thick paste (Table 6). On the other hand, the trough viscosity was significantly correlated to taste $(r=0.5, p=0.05)$, performance of the flour based meal $(r=0.6, p=0.05)$ and the choice of the best accession as ranked by consumers ( $r=0.5, p=0.05)$ (Table 5).

Final viscosity had a positive correlation with "flour-based meal" texture $(\mathrm{r}=0.5, \mathrm{p}=0.05)$, taste $(r=0.5, p=0.05)$ and overall performance of the flour $(r=0.7, p=0.05)$. In addition, the final viscosity was significantly correlated to the overall performance $(r=0.5, p=0.05)$. On the other hand, Set back viscosity was positively related to "ease of mingling" ( $r=0.5$, $\mathrm{p}=0.05$ ) confirming the observation that increased viscosity makes food processing harder to accomplish (Table 5). Overall, the best performing accession for "flour-based meal" was UG120193, while the worst performing accession was UG130016. In terms of pre-consumption attributes, UG120124 was the best owing to its rapid drying, while UG120198 was good for mingling.

\subsection{Relationship between "boiled roots" acceptability and pasting properties}

Like for the flour acceptability parameters, boiled root acceptability parameters were also correlated to pasting prorties. Peak viscosity was linearly related to mealiness $(\mathrm{r}=0.7, \mathrm{p}$ $=0.05)$, cooking time $(\mathrm{r}=0.5, \mathrm{p}=0.05)$ and sensorial softness $(\mathrm{r}=0.5, \mathrm{p}=0.05)$. Cassava accessions UG120124, UG120193, and UG120198, characterized with higher peak viscosity were considered mealy by consumers. Trough viscosity on the other hand, was positively correlated with cooking time $(\mathrm{r}=0.4, \mathrm{p}=0.05)$, mealiness $(\mathrm{r}=0.5, \mathrm{p}=0.05)$, softness $(r=0.6, p=0.05)$ and the overall performance of boiled roots $(r=0.6, p=0.05)$. Like peak viscosity, breakdown viscosity was positively related to cooking time $(r=0.5, p=0.05)$, mealiness $(r=0.7, p=0.05)$, softness as consumer perception $(r=0.5, p=0.05)$ and fibrousness $(\mathrm{r}=0.4, \mathrm{p}=0.05)$. 
Table 6: Correlation analysis showing the relationship between pasting properties and flour performance properties

\begin{tabular}{|c|c|c|c|c|c|c|c|c|c|c|c|c|}
\hline & Ease_mingling & Ease_drying & Stickiness & Taste & Texture & Perf_flour & Mealiness & $\begin{array}{l}\text { Cooking } \\
\text { time }\end{array}$ & Softness & Fibrousness & Perf._boiled & Perf_best \\
\hline Peak_viscosity & -0.2 & 0.3 & $0.5^{* *}$ & 0.2 & 0.0 & $0.5^{* *}$ & $0.7^{* *}$ & $0.5^{* *}$ & $0.5^{* *}$ & 0.1 & 0.3 & 0.3 \\
\hline Trough_viscosity & -0.1 & 0.3 & $0.4^{* *}$ & $0.5^{* *}$ & $0.4^{* *}$ & $0.6^{* *}$ & $0.5^{* *}$ & $0.4^{* *}$ & $0.6^{* *}$ & 0.3 & $0.6^{* *}$ & $0.5^{* *}$ \\
\hline Breakdown_viscosity & -0.1 & 0.2 & $0.4^{* *}$ & 0 & -0.2 & 0.3 & $0.7^{* *}$ & $0.5^{* *}$ & $0.3^{* *}$ & $0.4^{* *}$ & $0.4^{* *}$ & 0.2 \\
\hline Final_viscosity & 0.0 & 0.3 & 0.3 & $0.5^{* *}$ & $0.5^{* *}$ & $0.7^{* *}$ & 0.1 & $0.4^{* *}$ & 0.2 & 0.2 & 0.1 & $0.5^{* *}$ \\
\hline Setback_viscosity & $0.6^{* *}$ & 0.0 & $-0.4^{* *}$ & 0.1 & 0.3 & 0.1 & 0.2 & 0.1 & 0.2 & -0.1 & 0.2 & 0.2 \\
\hline Peak_time & -0.2 & 0.1 & -0.1 & 0.1 & 0.3 & 0.1 & 0.3 & 0.1 & 0.2 & 0.0 & 0.1 & 0.0 \\
\hline Pasting_temperature & 0.0 & -0.1 & -0.2 & 0.1 & 0.1 & 0.2 & 0.2 & 0.2 & 0.1 & 0.0 & 0.1 & 0.1 \\
\hline
\end{tabular}

${ }^{* *}$ Correlations significant at $5 \%$ level of significance

\section{Preprints (uruw.preprints.org) | NOT PEER-REVIEWED | Posted: 23 Mharch 2021}




\subsection{Discussion:}

Flour and boiled root acceptability in TRICOT based studies were studied. The TRICOT methodology allowed for a community led intervention, where farmers could also be engaged in the proper management procedures for producing standard products from cassava. Based on the results, differences in preferences were observed across study locations. The observed differences in study locations have been observed in related studies[6] and indicate the need for location specific breeding targets [3]. It also indicated that breeding programs need to provide accompanying packages detailing the processing and utilization of different cassava varieties.

The observed location based differences in moisture content pointed to differences in flour processing methods among different cassava growing communities[19]. Besides environmental differences in such locations may influence moisture contents given the hygroscopic nature of cassava flour [14]. This further underlines the need for location specific processing strategies to deliver standard cassava products. Location based differences were also observed for root softness. The observation could be attributed to environmental effects on cassava root properties with specific differences in soil factors influencing root properties [20].

The variability observed for root softness in most varieties however pointed to the possibility of using root softness as reliable metric to undertake selection in cassava [2]. However, this should be coupled to explainable variations between varieties as was the case in this study. More importantly, clones UG120198 and UG120024 which had low softness scores could be selected since farmers in Uganda are interested in varieties that have softer roots [7].

Location based differences in acceptability of the cassava product were observed in this study. The differences were expected given the different socioeconomic contexts in utilisation of cassava as a crop[21]. But most importantly, the study was able to identify accessions UG120193 and UG120156 as replacement varieties for the bench mark variety NAROCASS 1. This was based on the higher or equal to NAROCASS 1 performance displayed by these accessions in terms of their boiled root and flour meal properties. Such performance therefore warrants further advancement for possible release.

The significant differences $(\mathrm{p}<0.05)$ observed for final viscosity among the accesions shows that final viscosity can be used as a metric to define extent of starch retro-gradation. This is based on the differences in amylose molecular re-association [15] during cooling which leads to formation of viscous pastes on cooling. The differences therefore are related to the intercation between amylose content and other flour compistes and are measured as the setback viscosity[15][16]. Some accessions with higher peak time were observed such as UG120198 which indicate extended times for forming pastes and hence longer processing times[22]. The peak may thus influence the cooking time of boiled roots or the time taken to produce the cassava based meal much as it may not be the direct measure of cooking time[16].

The measurement of viscosity parameters provided for a clear understanding of some of the consumer based preferences of both the flour and boiled root based meals. Peak viscosity was found to be indicative of the stickness of the bread where highly viscous pastes were also sticky in nature. This was indicated by the positive significant relationships $(\mathrm{r}=0.5, \mathrm{p}=0.05)$ observed between peak viscosity and flour based meal stickiness. The positive significant relationships $(\mathrm{r}=0.6, \mathrm{p}=0.05)$ observed between performance of the flour based meal and trugh viscosity indicated that performance of the flour based meal was perceived as a processing property[1][7] by the consumers and 
thus could be easily measured from the ability of the paste not to "collapse" during preparation/cooking. The high positive and significant relationship observed between final viscosity and flour meal over all performace is due to the fact that the "flour-based meal" is consumed on cooling[7]. Therefore, final consumer perception is very likely to be influenced by the final viscosity.

Table 5: Relationship between pasting properties of flour obtained from different accessions

\begin{tabular}{|c|c|c|c|c|c|c|c|}
\hline & $\begin{array}{l}\text { Peak_ vis- } \\
\text { cosity }\end{array}$ & $\begin{array}{l}\text { Trough } \\
\text { _viscosity }\end{array}$ & $\begin{array}{l}\text { Breakdown } \\
\text { _viscosity }\end{array}$ & $\begin{array}{l}\text { Final_ vis- } \\
\text { cosity }\end{array}$ & $\begin{array}{l}\text { Setback } \\
\text { _viscosity }\end{array}$ & $\begin{array}{l}\text { Peak } \\
\text { time }\end{array}$ & $\begin{array}{l}\text { Pasting - tem- } \\
\text { perature }\end{array}$ \\
\hline Peak_viscosity & 1 & & & & & & \\
\hline $\begin{array}{l}\text { Trough } \\
\text { _viscosity }\end{array}$ & $0.7^{* *}$ & 1 & & & & & \\
\hline $\begin{array}{l}\text { Breakdown } \\
\text { _viscosity }\end{array}$ & $0.9^{* *}$ & 0.3 & 1 & & & & \\
\hline Final_viscosity & $0.6^{* *}$ & $0.9^{* *}$ & 0.2 & 1 & & & \\
\hline $\begin{array}{l}\text { Setback } \\
\text { _viscosity }\end{array}$ & $-0.5^{* *}$ & -0.2 & -0.3 & 0.1 & 1 & & \\
\hline Peak_time & -0.1 & $0.4^{* *}$ & $-0.6^{* *}$ & -0.2 & -0.2 & 1 & \\
\hline $\begin{array}{l}\text { Pasting_ tem- } \\
\text { perature }\end{array}$ & $-0.5^{* *}$ & -0.2 & $-0.5^{* *}$ & -0.1 & 0.2 & $0.4^{* *}$ & 1 \\
\hline
\end{tabular}

${ }^{* *}$ Correlations significant at $5 \%$ level of significance

For the boiled root based meal, peak viscosity was significantly related to boiled root mealiness. Mealiness is an essential attribute preferred for boiled cassava roots[7][3]. Ironically, it's difficult to quantify and henceforth breed for[7]. Findings presented herein suggest that high peak viscosity can within limits be used to infer a better degree of mealiness.

However, some pasting parameters were not definitive of any consumer preference based characteristics. For example, there was no relationship observed between breakdown viscosity and consumer acceptability attributes. It suffices to note that break down viscosity defines paste stability during the mingling process[9], a phenomenon that may not be adequately assessed from the final flour product, "flour-based meal". Therefore, breakdown viscosity could not be used to inform selection decisions. In addition, it calls for possible assesement of these traits by the consumer even through the mingling process where possible. Since breakdown viscosity is related positively and significantly related to peak viscosity $(\mathrm{r}=0.9)$ and other pasting properties (Table 6), inferences for consumer based understanding can be based on peak viscosity as a metric.

The results obtained in this study further suggest that softness-related parameters which also include mealiness, could be an indirect measure of stability of root components after boiling[7]. This is further supported by the positive correlation between final viscosity and overall performance of "boiled root" $(\mathrm{r}=0.6, \mathrm{p}=0.05)$, since a more viscous and stable matrix in the root would influence the observed softness score. Negative correlations were observed between pasting temperature and cooking time $(r=-0.5, p=0.05)$, mealiness $(\mathrm{r}=-0.5, \mathrm{p}=0.05)$ and overall performance of boiled $\operatorname{root}(\mathrm{r}=-0.4, \mathrm{p}=0.05)$. This shows that higher pasting times result from increased time for processing the root a factor that is inversely related to good root performance. Such relationships reveal that pasting 
properties have important associations with cassava root quality attributes as perceived by consumers. The findings also point to the possibility of using pasting properties for a better description of the sensorial properties of cassava root and flour based meals.

\subsection{Conclusions}

86 87

From the acceptability analyses undertaken, accession UG120193 was the most acceptable accession among the accessions tested. However comparative acceptability was observed for NAROCASS 1, UG120156 and UG130007. These accessions had comparatively high peak viscosity $(\sim 5500 \mathrm{cP})$ and moderate peak times of 4.2 minutes showing that they had similar pasting behavior. Thus acceptability can in part be explained by the pasting behavior of flour from different varieties. Pasting properties were linearly related to some boiled root properties such as mealiness, cooking time and softness. These pasting properties can be used to understand root based manifestations of acceptability after boiling and hence selection of varieties with acceptable boiled roots. From the study, it was observed that according to farmers, the most acceptable accession over all was UG120193 followed by UG120156. The two accessions performed equally to or higher than NAROCASS 1 and can therefore be considered as replacement varieties for the bench mark variety in this case. Pasting properties and viscosity parameters related to the processing of cassava flour included the peak viscosity, trough viscosity, and the final viscosity. These are relevant metrics for assessing the acceptability of the boiled root and flour based meal. Thus they can also be used to define theacceptability of particular varieties for flour based meal production.
90

91

92

93

94

95

96

97

98

99

100

101

102

103

104

105 
[11] E. Nuwamanya, Y. Baguma, N. Emmambux, J. Taylor, and R. Patrick, "Physicochemical and functional characteristics of cassava starch in Ugandan varieties and their progenies," J. Plant Breed. Crop Sci., vol. 2, no. 1, pp. 1-11, 2010.

[12] P. Iragaba et al., "Estimates for heritability and consumer-validation of a penetrometer method for phenotyping softness of cooked cassava roots," African Crop Sci. J., vol. 27, no. 2, p. 147, 2019.

[13] Y. Park, “Moisture and Water Activity," Handb. Process. Meats Poult. Anal., no. December, pp. 35-67, 2008.

[14] R. C. Chisté, J. M. Cardoso, D. A. da Silva, and R. da S. Pena, “Hygroscopic behaviour of cassava flour from dry and water groups," Ciência Rural, vol. 45, no. 8, pp. 1515-1521, 2015.

[15] M. Winger, H. Khouryieh, F. Aramouni, T. Herald, and M. W. E. T. Al, “Gluten-Free Flour Tortilla," vol. 37, pp. 95-106, 2014.

[16] R. Ezekiel, G. Rana, N. Singh, and S. Singh, "Physico-chemical and pasting properties of starch from stored potato tubers," J. Food Sci. Technol., vol. 47, no. 2, pp. 195-201, 2010.

[17] A. G. Kulamarva, V. R. Sosle, and G. S. V. Raghavan, “Nutritional and rheological properties of sorghum,” Int. J. Food Prop., vol. 12, no. 1, pp. 55-69, 2009.

[18] G. Justamante Händel Schmitz et al., "Selected physicochemical properties of starches isolated from ten cassava varieties reveal novel industrial uses," Starch/Staerke, vol. 69, no. 7-8, 2017.

[19] A. Abass, P. Amaza, B. Bachwenkizi, K. Wanda, A. Agona, and N. Cromme, "The impact of mechanized processing of cassava on farmers' production efficiency in Uganda," Appl. Econ. Lett., vol. 24, no. 2, pp. 102-106, 2017.

[20] A. A. C. Alves, “Cassava botany and physiology.," Cassava Biol. Prod. Util., pp. 67-89, 2009.

[21] T. Buyinza and L. Kitinoja, Commodity Systems Assessment of Cassava in Uganda, vol. PEF White, no. August. 2018.

[22] A. U. Mohammed, K. B. Mathew, and J. Samaila, “Functional properties of weaning food blends from selected sorghum (Sorghum bicolor (L.) Moench) varieties and soybean (Glycine max),” African J. Food Sci., vol. 10, no. 8, pp. 112-121, 2016. 